\title{
Bibliometric Analysis of Nursing Informatics Research
}

\author{
Jialin LIU ${ }^{\mathrm{a}, 1}$, Siru LIU ${ }^{\mathrm{b}}$, Qingke SHI ${ }^{\mathrm{a}}$ and Miye WANG ${ }^{\mathrm{a}}$ \\ ${ }^{a}$ Department of Medical Informatics, West China Hospital, Sichuan University, China \\ ${ }^{\mathrm{b}}$ Department of Biomedical Informatics, University of Utah, Salt Lake City, Utah, USA
}

\begin{abstract}
This study aims to provide a bibliometric overview of research at nursing informatics and understand the state in nursing informatics in the last ten years. We used the Web of Science to extract relevant literature published from 2009 to 2018. A total of 455 articles were retrieved and analyzed. The total of the top 5 institutions, countries, journals was discussed. This study will help researchers to understand trends and the situation in nursing informatics research.
\end{abstract}

Keywords. Nursing informatics, bibliometric, analysis

\section{Introduction}

Nursing informatics is important to all nursing specialty [1]. The purpose of the study is to offer a macroscopic overview of the main characteristics of nursing informatics articles based on bibliometric analysis. The analysis will help researchers understand the publication of papers dynamics and trends and prolific authors, institutions, and countries in the field.

\section{Methods}

The two reviewers independently searched articles in Web of Science core collection (WoSCC) period from January 1, 2009, to December 31, 2018, with the topic term "nursing informatics." Only articles, proceeding papers, and reviews were included. Articles published in non-English languages were also excluded.

\section{Results}

Of the 982 articles originally collected, 480 unrelated articles were excluded. 15 articles published in non-English and 32 articles of other types were removed with the WoS tool. Finally, we identified and analyzed 455 articles (Figure 1). Of the 455 articles retrieved include original articles $254(55.82 \%)$, proceeding papers $186(40.88 \%)$, and reviews 15 $(3.30 \%)$.

\footnotetext{
${ }^{1}$ Corresponding Author, Jialin Liu, Department of Medical Informatics, West China Hospital, Sichuan University, Chengdu, Sichuan, China; E-mail: dlj18@163.com.
} 


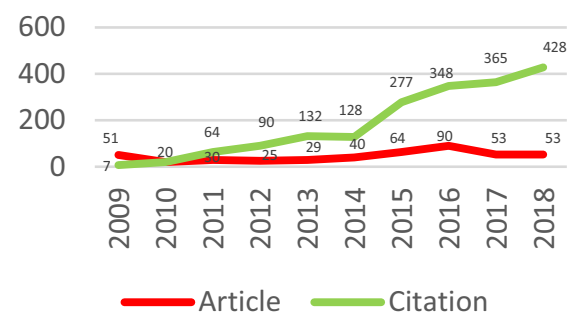

Figure 1. Annual distribution of publications and citation

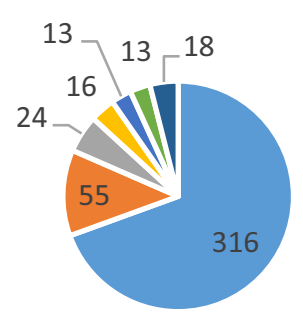

Figure 2. Journal publications

\subsection{Journals Publishing on Nursing Informatics}

In total, 455 articles on nursing informatics were published in 108 different journals. Of the 108 journals, 55 journals (47.83\%) published only one article. However, the Studies in Health Technology and Informatics published 316 articles (69.45\%) on nursing informatics. Figure 2 shows a list of articles the top 5 journals in the field of nursing informatics research.

\subsection{Institutions and Countries}

The 455 articles originate from 52 different countries/regions and 572 institutions. Table 1 shows the top 5 productive institutions/organizations and countries/regions on nursing informatics research.

Table 1. Top 5 of most productive institutions and countries publishing on nursing informatics.

\begin{tabular}{llcl}
\hline institutions /Organizations & Record & Countries/Regions & Record \\
\hline Univ Sao Paulo (Brazil) & $30 / 6.60 \%$ & USA & $210 / 46.15 \%$ \\
Univ Minnesota (USA) & $22 / 4.84 \%$ & Brazil & $77 / 16.92 \%$ \\
Univ Victoria (Canada) & $21 / 4.62 \%$ & Canada & $45 / 9.89 \%$ \\
Columbia Univ (USA) & $19 / 4.18 \%$ & Australia & $40 / 8.79 \%$ \\
Nat Yang Ming Univ (Taiwan) & $12 / 2.64 \%$ & Taiwan & $28 / 6.15 \%$ \\
Univ Fed Santa Catarina (Brazil) & $12 / 2.64 \%$ & & \\
\hline
\end{tabular}

\section{Conclusion}

The Studies in Health Technology and Informatics is the most important source of nursing informatics literature. The University of Sao Paulo (Brazil) is the most productive institution in nursing informatics, followed by the University of Minnesota and the University of Victoria. The United States clearly leads the ranking obtaining the most significant results in the number of nursing informatics papers. 


\section{References}

[1] McGonigle D, Hunter $\mathrm{K}$, Sipes $\mathrm{C}$ et al. Why nurses need to understand nursing informatics. AORN,100(2014):324-327. 